DE GRUYter DOI: 10.1515/plass-2016-0008

Naser Sabaghnia*, Mohsen Janmohammadi

Department of Agronomy and Plant Breeding, Agriculture College, University of Maragheh, Iran."Corresponding Author’s e-mail sabaghnia@maragheh.ac.ir

\title{
BIPLOT ANALYSIS OF SILICON DIOXIDE ON EARLY GROWTH OF SUNFLOWER
}

\begin{abstract}
Research into nanotechnology has advanced in almost all fields of technology and the aim of this study was to evaluate the role of nano-silicon dioxide (nano- $\left.\mathrm{SiO}_{2}\right)$ in germination performance sunflower. Germination and seedling growth are the most important stage of plant development and are critical factors to crop production and are essential to achieve optimum performance. The effects of pre-germination hydration in solutions of nano-SiO2 $(0,0.2,0.4,0.6,0.8,1$ and $1.2 \mathrm{mM}$ for $8 \mathrm{~h})$ on germination characteristics of sunflower were investigated. The trait by treatment (TT) biplot explained $93 \%$ of the total variation of the standardized data ( $77 \%$ and $16 \%$ for the first and second principal components, respectively). According to polygon-view of TT biplot, T2 $(0.2 \mathrm{mM})$ had the highest values for all of the measured traits except mean germination time and the time to $50 \%$ germination. The germination percentage was determined as the best trait and showed the high association with promptness index, energy of germination and germination rate traits. The results of the present study indicated that pre-sowing seed treatments with low concentration of nano-SiO $\mathrm{Sad}_{2}$ favorable effect sunflower seed germination and seedling early growth. Such a similar outcome could be applied in the future to outline other crops in response to nano-particles as well as to help define tolerance tools for recommendations in stressful conditions in the world.
\end{abstract}

Key words: germination rate, nano-sized materials, seed priming, TT biplot

\section{INTRODUCTION}

Sunflower (Helianthus annuus L.) is important oilseed crops in the world and it is arousing the interest of producers, due to the possibility of using its oil as human food or as raw material for industrial proposes. Its growing area has been very variable and ranged from 110000 to 30000 hectares in Iran in last two decades (1990-

Communicated by Grzegorz Żurek 
2010), but at recent years its cultivation areas is increased to 68000 hectares (FAOSTAT 2012). It has been estimated that world production of sunflower is exceeded 37 million tones from 25 million hectares cultivated areas (FAOSTAT 2012). Sunflower is primarily grown for edible oil, but also as a raw material for the processing industry, livestock feed and beekeeping proposes. In the recent few years, sunflower oil is used for industrial biodiesel production. It is harvested early in the fall for providing enough time for planting winter cereal crops and plays an important role in crop rotation systems (Mijic et al. 2009).

Poor crop establishment was identified as one of the major problems in crop production under arid and semi-arid regions (Harris et al. 1999). It seems that seed priming would improve seed germination and plant growth with adjusting the hydration level within seeds which permits seedlings to emerge more rapidly and to facilitate the uniform seedling stand (BASRA et al. 2006). Most priming treatments involve imbibing seed with constrained amounts of water to allow adequate hydration and progress of biochemical processes but hindering the protuberance of the radicle (Ashraf and Foolad 2005). In recent years, the progress of nanotechnology has appreciably increased the utilization domain of nano-particles in agriculture and some studies indicated the potential of nano-particles in improving seed germination and seedling early growth (Siddiqui \& Al-whaibi 2014; Sabaghnia and Janmohammadi 2014). Nano-particles can easily enter into plant by overcoming the cell wall barrier in comparison with bulk materials, but their behavior inside the plant system is unpredictable (Nair et al. 2011).

The correlation coefficients are important issues in determining the degree of association of different germination properties. This analysis is a reliable statistical method, which provides tool to quantify the associations among different traits Effective interpretation of the data is important at all stages of plant evaluation and several methods have been used in exploration for an understanding of the data structure. These methods may differ in overall appropriateness; different methods usually lead to the similar conclusions for a given dataset. Yan et al. (2000) have developed a genotype main effect plus genotype by environment (GGE) biplot methodology for the graphical analysis of multi-environment trial data. This method can be used for all types of two-way dataset such as treatments with multiple traits. Dehghani et al. (2008) have used a treatment by trait (TT) biplot, which is an application of the GGE biplot to study the treatment by trait data.

Silicon as the second most abundant mineral element in the soil has been recognized as a beneficial nutrient for plant growth and development (Liang et al. 2007). It appears that silicon have an important function in plant protection because it deposit on the walls of epidermis and vascular tissues and play role as a physicomechanical barrier, (Ma and Yamaji 2008), and also regulate some physiological process related to defense mechanisms in plants (Bao-Shan et al. 2004). It seems that, seed prehydration in solution of nano-silicon can be considered as a seed vigour enhancement treatment. The pervious studies revealed that some advantages are associated with priming treatments for seed vigor improvement of some crops, but 
there is dearth of information about the germination performance of nano-particles primed achenes of sunflower. Therefore it was essential to evaluate the application of nano-silicon in order to improve sunflower germination capability. The purpose of this research was to evaluate the effect seed priming in nano-silicon on seed germination performance of sunflower to find out the most promising concentration using biplot statistical tool.

MATERIALS AND METHODS

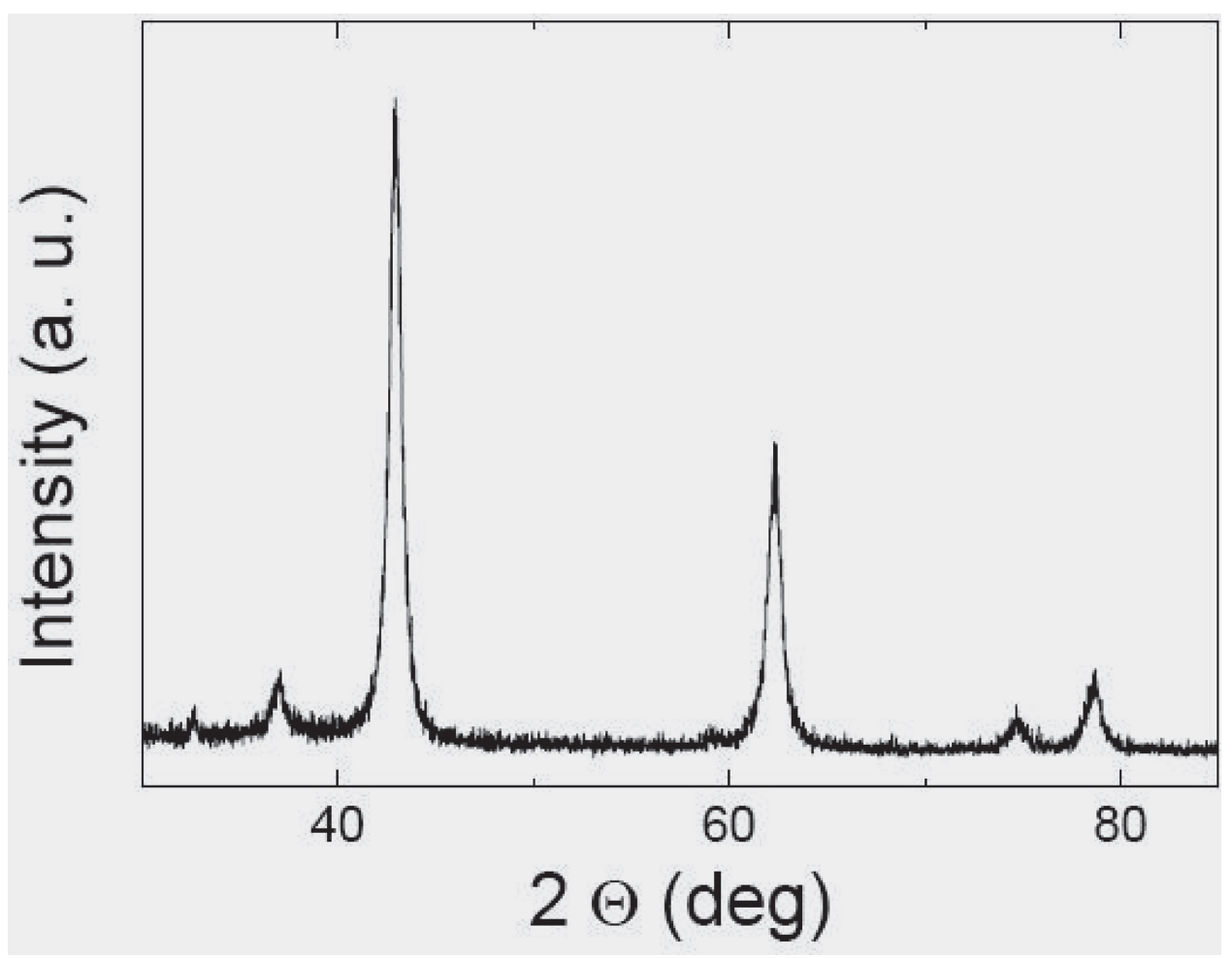

Fig. 1. X-Ray diffraction pattern of $\mathrm{SiO} 2$ nano-particles.

Seeds of sunflower (cultivar Azargol) were immersed in a 5\% sodium hypochlorite solution for $10 \mathrm{~min}$ to ensure surface sterility hen washed with distilled water for three times. Pre-hydration treatments were including control (non-primed seeds) and seeds soaking in solutions containing different concentrations of nano-silica $\left(0.2,0.4,0.6,0.8,1\right.$ and $1.2 \mathrm{mM}$ for $8 \mathrm{~h}$ ). Nano-particles of $\mathrm{SiO}_{2}$ were prepared from the Pishgaman-Nano, Iran. The particle sizes of $\mathrm{SiO}_{2}$ ranged from 20 to $30 \mathrm{~nm}$ and specific surface area of nano-sized silicon was $180-600 \mathrm{~m}^{2} \times \mathrm{g}^{-1}$ and purity was $99.7 \%$. The result of X-ray analysis of $\mathrm{SiO}_{2}$ (Fig. 1) and the high-resolution transmission electron microscopy (HRTEM) image of the nano-particle sample (Fig. 2) 
are shown. After presoaking, all seeds were washed with distilled water then allowed to air dry for $48 \mathrm{~h}$ at $25^{\circ} \mathrm{C}$ (Iqbal \& Ashraf 2007). The experiment was carried out based on completely randomized design with three replications. Of 30 primed seeds were placed on $9 \mathrm{~cm}$ petri dishes among papers and wetted with $10 \mathrm{ml}$ sterilized distilled water.

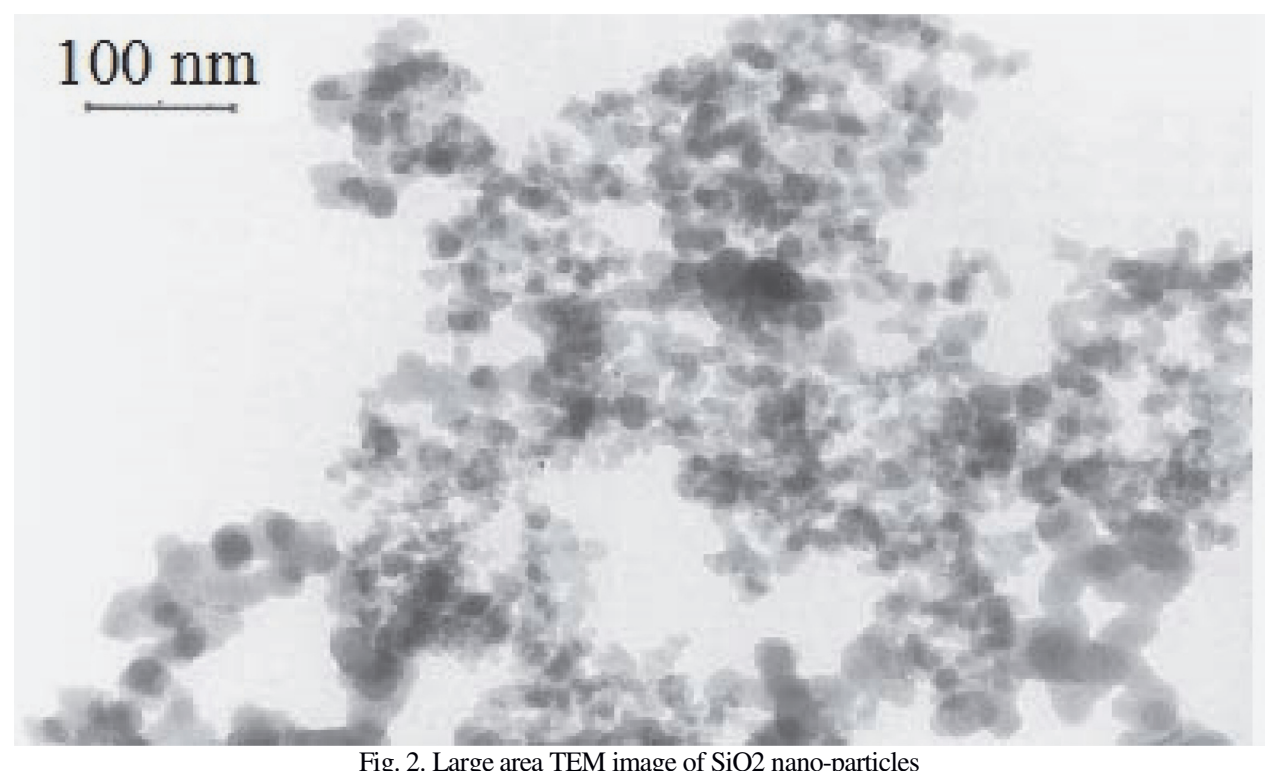

The experiment is performed in a dark incubator at $20 \pm 1^{\circ} \mathrm{C}$ and germinated seed were recorded daily for eight days. Germination was considered to have occurred when the seed developed at least $2 \mathrm{~mm}$ long radical. The final germination percentage was calculated based on total number of germinated seeds at the end of tenth day. The all evaluations were executed as described in Seedling Evaluation Handbook (AOSA, 1991). Mean germination time (MGT) was computed according to ELLIS \& ROBERTS (1981) and germination rate (GR) considered as the reciprocal of the mean germination time (Ranal \& Santana 2006). The time to 50\% germination (T50) was calculated according to the following formula of Farooq et al. (2005) and energy of germination (EG) was calculated four day after start of the experiment. It is the percentage of germinating seeds 4 days after planting relative to the total number of seeds tested (RUAN et al. 2002). Promptness index (PI) according to Ashraf et al. (2006). The fresh weight of root and shoot of seedlings was determined by weight of the root and shoot separately on electric balance. After the fresh weight taken then the seedlings was kept in a hot air oven at $60^{\circ} \mathrm{C}$ for $48 \mathrm{hrs}$ then the weight of dry matter was recorded.

Because the traits were measured in different units, the biplots were generated using the standardized values of the traits means using GGEbiplot software (YAN 2001). Up-to-date information on GGE biplot and new version of GGEbiplot package are available at http://www.ggebiplot.com. The biplot analysis was according to 
Model 2 (Transform=0) within-trait standard deviation standardized (Scale=1), and trait-centred (Centering=2). The polygon views were based on cultivar-focused singular value partitioning ( $\mathrm{SVP}=2$ ), while the vector views were based on the traitfocused singular value partitioning and is, therefore, appropriate for visualizing the relationships among traits and cultivars. The cultivar by trait (GT) biplot model for cultivar by trait interaction biplot analysis was used according to Yan \& Rajcan (2002). The treatment by trait (GT) biplot model equation (Yan \& Rajcan 2002) is presented as follows:

$$
\frac{\alpha_{i j}-\beta_{j}}{\sigma_{j}}=\sum_{n=1}^{2} \lambda_{n} \xi_{i n} \eta_{j n}+\varepsilon_{i j}=\sum_{n=1}^{2} \xi_{i n}^{*} \eta_{j n}^{*}+\varepsilon_{i j}
$$

where $\alpha_{i j}$ is the mean value of treatment $i$ for trait $j, \beta_{j}$ is the mean value of all treatments in trait $\mathrm{j}, \sigma_{y}$ is the standard deviation of trait $\mathrm{j}$ among the treatment means, $\lambda_{n}$ is the singular value for principal component $n(\mathrm{PCn}), \xi_{\text {in }}$ and $\eta_{j n}$ are scores for treatment $\mathrm{i}$ and trait $\mathrm{j}$ on PCn, respectively, and $\varepsilon_{i j}$ is the residual associated with treatment $i$ in trait $j$. To achieve symmetric scaling between the treatment scores and the trait scores the singular value $\lambda_{n}$ has to be absorbed by the singular vector for treatments $\xi$ in and that for traits $\eta_{j n}$ and $\eta_{j n}^{*}=\eta_{j n} \times \lambda_{n}^{0.5}$. Because of $\mathrm{n}=2$, only PC1 and PC2, are retained in the model and such a model tends to be the best for extracting pattern and rejecting noise from the data.

\section{RESULTS}

The TT biplot for sunflower dataset based on the method described by Yan \& Rajcan (2002), explained 93\% of the total variation of the standardized data. This high percentage reflects the relatively moderate complexity of the relationships among the measured traits. The first two principal components (PC1 and PC2) explained $77 \%$ and $16 \%$, respectively. In the TT biplot, a vector is drawn from the biplot origin to each marker of the tester (treatment) to facilitate visualization of the relationships between and among the measured traits as well as treatments. According to Rubio et al. (2004), Dehghani et al. (2008) and Sabaghnia et al. (2011), the basic structure among the traits should be captured by the biplots. The polygon view of the TT biplot aids identifies entries (treatments) with the highest values for one or more traits. For this target, the genotypes that are connected with straight lines so that a polygon is formed with all other traits contained within the polygon. The biplot in Fig. 3A presents the data of seven treatments in nine different traits and the following information can be obtained: the vertex treatments in this investigation are T2, T3, T6 and T7. These treatments are the best or the unsuitable treatments in some or all of the traits since they had the longest distance from the origin of biplot. Therefore, $\mathrm{T} 2 \mathrm{had}$ the highest values for all of the measured traits except 
MGT and T50 (i.e. RDW, SDW, SL, PI, GP, EG and GR). The T2 and the other treatments (in this case: T4) of this sector had suitable amounts for the above mentioned traits (Fig. 3A). The vertex treatment T6 was suitable for MGT while the vertex treatment $\mathrm{T} 7$ and its related treatments ( $\mathrm{T} 1$ and $\mathrm{T} 5$ ), which fall in its sector, were suitable for T50 (Fig. 3A). The other vertex treatment (T3) and related sector was not suitable performance for the measured traits (Fig. 3A).

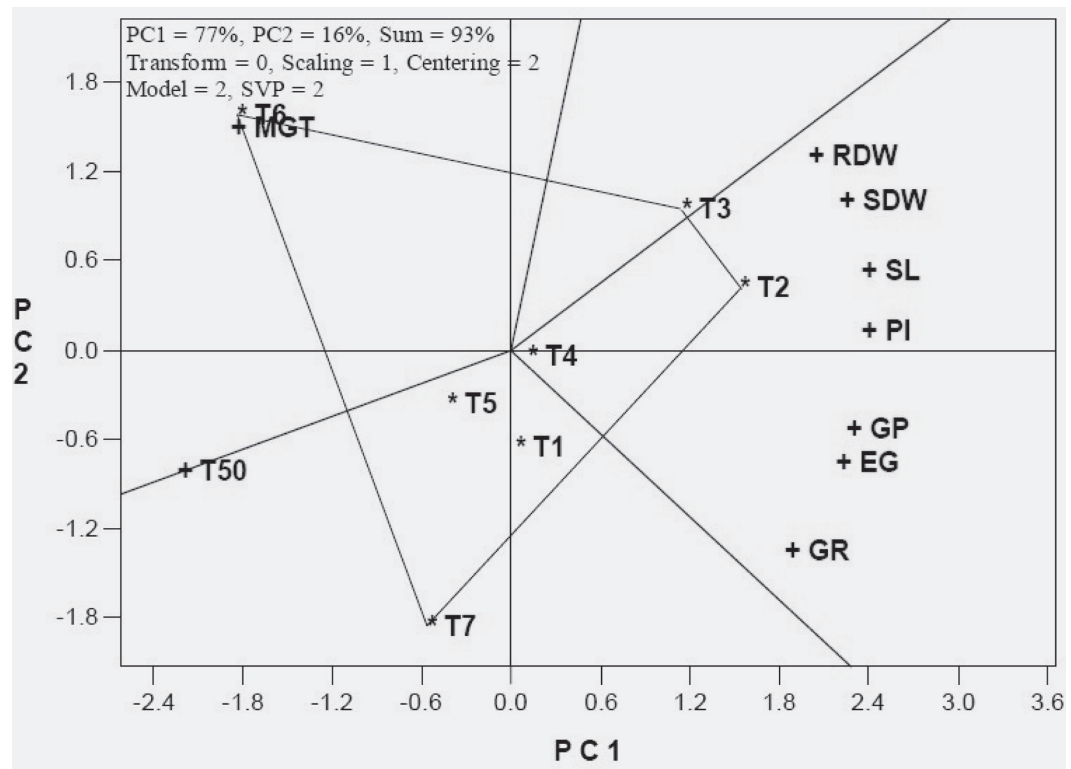

A

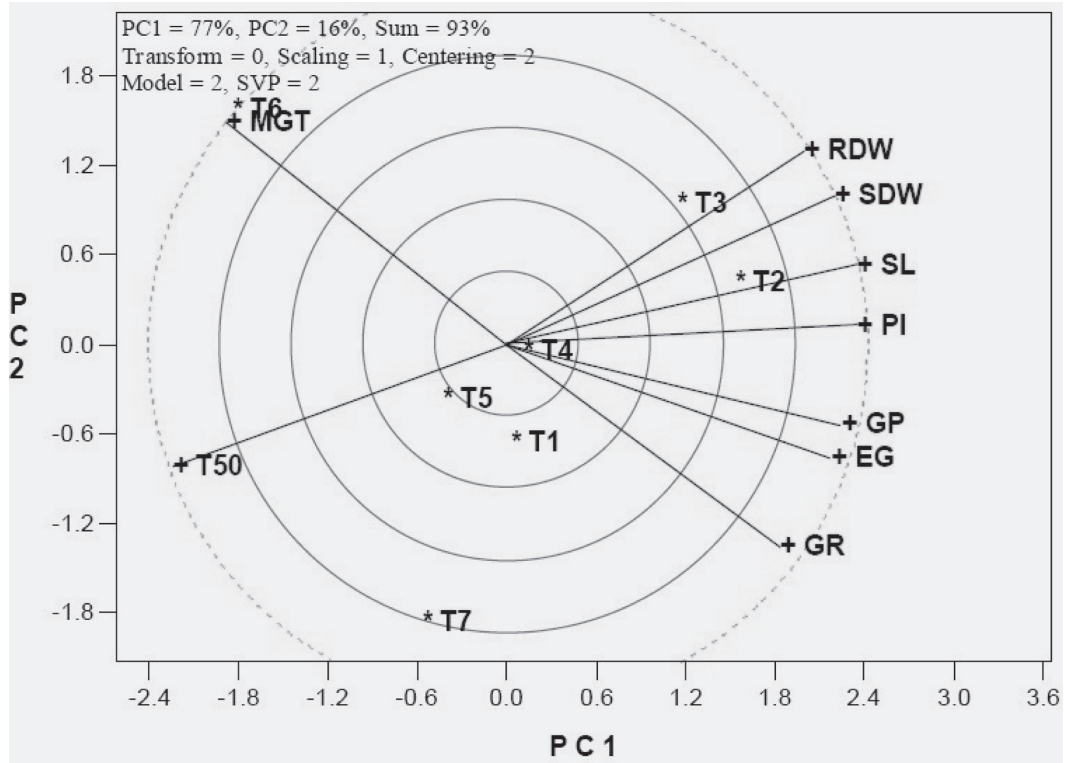

B

Fig. 3. (A) Polygon view of TT biplot showing which treatment had the highest values for which traits and (B) vector view of TT biplot, showing the interrelationship among all measured traits of sunflower 


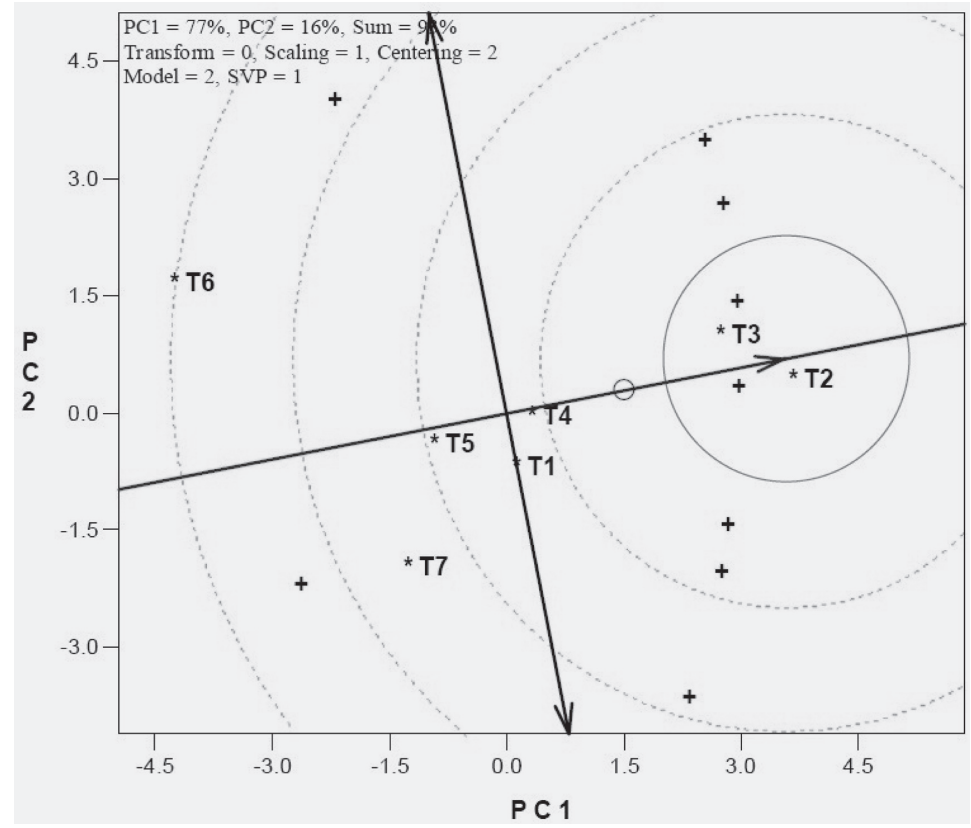

A

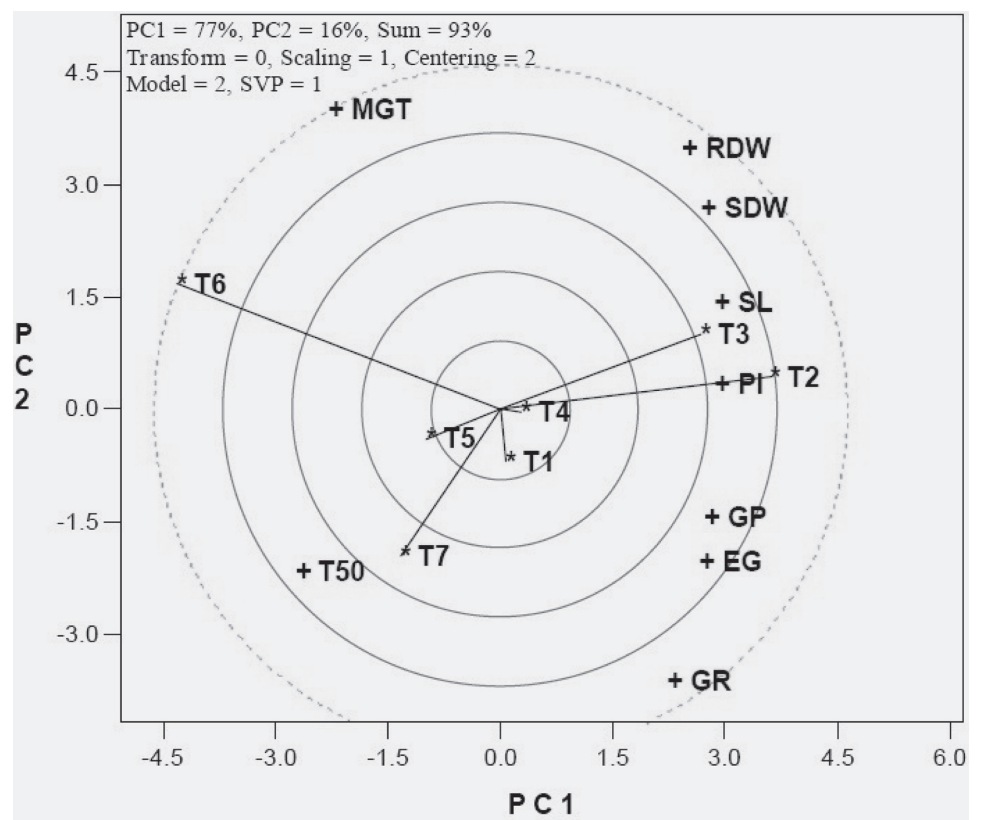

Fig. 4. (A) vector view of TT biplot, showing the interrelationship among all treatments and (B) ideal entry view of TT biplot, showing the relationships of treatments with ideal entry in sunflower 
Provided that the biplot model described a sufficient amount of the total variation (in this case, 93\%), the correlation coefficient between any two traits is approximated by the cosine of the angle between their vectors (Yan \& Rajcan 2002). The correlation coefficients among the traits indicate that the biplot currently shows relationship among the traits that had relatively large loading on both $\mathrm{PC} 1$ and PC2. Therefore, the most prominent relations by Fig. 3B are: a strong positive correlation between RDW and SDW; between SL and PI; and between GP and EG; as indicated by the small obtuse angles between their vectors $(r=\cos 0=+1)$. The correlations between RDW and GR; RDW and MGT; and T50 and GR were near zero (Fig. 3B) as indicated by the near perpendicular vectors $(r=\cos 90=0)$. There was a negative correlation between GR and MGT, and between RDW and T50 (Fig. 3B) as indicated by the near angle of approximately 180 degrees $(\mathrm{r}=$ $\cos 180=-1)$. Most of the mentioned results can be verified using original correlation coefficients, but less of them can not be verified using original correlation coefficients, because the biplot accounted for $<100 \%$ of the total variation (93\%). The statistical properties of this method have been described in detail by Yan et al. (2000) and Yan \& Rajcan (2002).

Similar to tester vector-view biplot, the most prominent relations by entry vector-view biplot (Fig. 4A) are: a strong positive correlation between $\mathrm{T} 2$ and $\mathrm{T} 3$ as indicated by the small obtuse angles between their vectors ( $\mathrm{r}$ $=\cos 0=+1$ ). The correlation between $\mathrm{T} 2$ and $\mathrm{T} 7$ was near zero (Fig. 4A) as indicated by the near perpendicular vectors $(r=\cos 90=0)$. There was a negative correlation between $\mathrm{T} 2$ and $\mathrm{T} 6$, and between $\mathrm{T} 3$ and $\mathrm{T} 7$ (Fig. $4 \mathrm{~A})$ as indicated by the near angle of approximately 180 degrees $(\mathrm{r}=$ $\cos 180=-1$ ). An ideal entry (treatment) has been defined as the entry that combines several suitable traits in it's' characteristics. Based on Fig. 4B, the ideal treatment is strongly related to as $\mathrm{T} 2$ and $\mathrm{T} 3$ treatments while treatment $\mathrm{T} 6$ following to treatments $\mathrm{T} 7$ and $\mathrm{T} 5$ were not suitable. Therefore, it seems that application of $\mathrm{SiO}_{2}$ nano-particles in low concentration $(0.2$ and $0.4 \mathrm{mM}$ ) would be more effective than moderate or high concentrations. Maybe, in high concentrations of $\mathrm{SiO}_{2}$ nano-particles, the toxicity of nanoparticles is more effective than its improving property.

Due to great importance of germination percentage (GP) in crop establishment of sunflower, this trait was compared with the other traits and visually displayed in Fig. 5. In the context of TT biplot, the GP was determined as the best trait, which showed the above average performance, was EG following to PI and GR. The other remaining traits including SL> SDW $>$ RDW $>$ T50 $>$ MGT indicated below performance (Fig. 5). Therefore it seems that, in germination percentage characteristic, energy of germination, promptness index and germination rate are important. In other word, the rate and power of seed germination are greatly related and influenced the germination percentage in sunflower. 


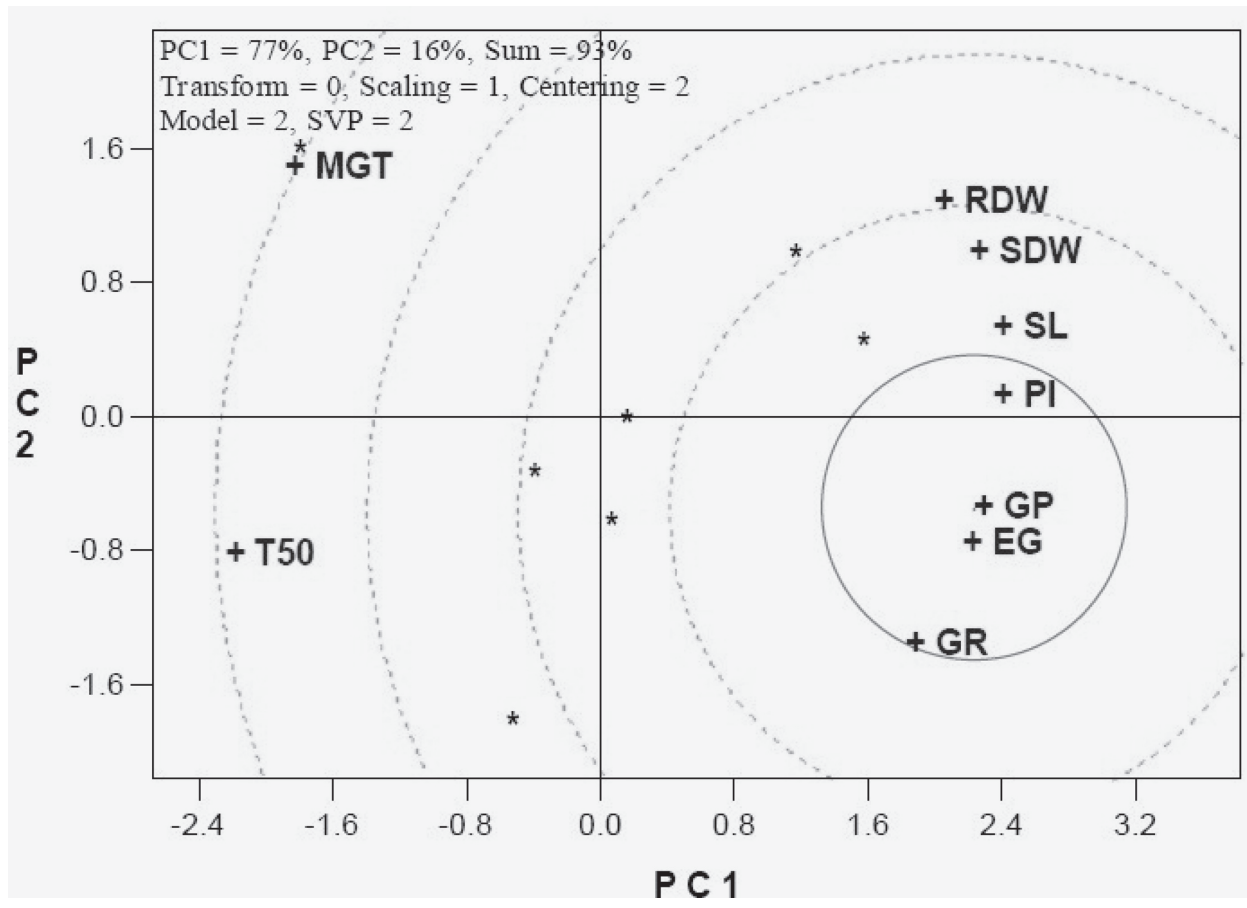

Fig. 5. Tester comparison view of TT biplot showing the relationships of traits with germination percentage (GP) in sunflower

\section{DISCUSSION}

Nanotechnology has emerged as a new field, and nano-particles have become a centre of attraction due to their unique properties compared to bulk materials. The $\mathrm{SiO}_{2}$ nano-particles could be acts as a delivering agent into plants but the mechanism of their action on plants is still scarce. Seed germination prepares a proper foundation for plant growth and yield performance. In the present study application of $\mathrm{SiO}_{2}$ nano-particles enhanced seed potential by increasing the related traits or parameters of seed germination in sunflower at low concentrations. Among the treatments, application of 0.2 and $0.4 \mathrm{mM}$ of $\mathrm{SiO}_{2}$ nanoparticles proved best by giving the highest values for most of the seed germination parameters. Also, our findings revealed that seed soaking in $\mathrm{SiO}_{2}$ nanoparticles solution resulted in vigour enhancement. These results confirmed the pervious study on tomato germination by utilization of nano-silicon dioxide (Siddiqui \& Al-Whaibi 2014).

This research demonstrated that TT biplot is a useful tool for the analysis of sunflower dataset. Based on TT biplots, the similarities and differences among traits and treatments and the similarities among the treatments from traits aspects can be visualized. The results of analysis by the TT biplot showed that 
treatments $\mathrm{T} 2$ and $\mathrm{T} 3$ showed better performance than other treatments tested in this experiment. These two treatments, in the present investigation, identified as the most consistent-performing treatments. The use of these treatments would be ensuring high performance in germination of sunflower with application of $\mathrm{SiO}_{2}$ nano-particles. Similar conclusions we can address about correlations among traits from TT biplot analysis. In germination percentage, some traits including energy of germination, promptness index and germination rate were important and influenced the germination percentage in sunflower from the the rate and power of seed germination aspects. The GT biplot analysis was shown to be more informative than conventional methods for distinguishing special features in treatments and this statistical method was found to be proper for test of treatments.

It appears that silicon has an important role in plant protection against biotic and abiotic stress (Ma 2004) and it has been reported that silicon application could alleviate the adverse effects of salinity stress on seed germination (Haghighi et al. 2012). The faster and synchronized seed germination might be attributed to enhanced metabolic activities in the primed achenes (Wahid et al. 2008). In the arid and semi-arid regions which are adverse with abiotic stresses, the rate or entirely hinder seed germination and seedling emergence in late spring and early summer are decreased. In this respect, the application beneficial nano-particles for pre-sowing treatments and utilization of seeds with enhanced vigor can be a practicable strategy to obtain healthy seedlings and a better crop stand under unfavorable environmental conditions.

It is clear that the TT biplot is an excellent statistical tool for visual data analysis. Compared with conventional statistical methods of data analysis, the TT biplot approach has some advantages including: (i) the graphical presentation of the dataset which greatly enhances researcher ability to understand the pattern of the data; (ii) the more interpretability which is facilitates pair-wise genotype comparisons; and (iii) facilitating the identification of possible sectors or groups of testers or entries (Yan \& Hunt 2001; Yan et al. 2007). A potential constraint of the biplot method is that it may fail to explain most of the variation and therefore fail to display all patterns of the data. This is most likely to occur with large datasets, but in our dataset $93 \%$ of total variation is explained by the two first PCs and this constraint was not observed. However, even when this is the case, it can be ensured that the biplot of PC1 versus PC2 still displays the most important linear patterns of the data (Yan and Hunt 2001). Also, other types of biplots, such as one consisting of PC3 versus PC4, may be required to fully understand the dataset's pattern.

Result emphasized that controlled imbibition of sunflower seeds in $\mathrm{SiO}_{2}$ nano -particles solution followed by dehydration could significantly enhance germination potential. It appears that treated seeds usually would exhibit rapid germination when absorb water under field conditions where the time from sowing to seedling establishment is of considerable importance. According to the results, 
it seems that the $\mathrm{SiO}_{2}$ nano-particles with the increased penetration power of the seed facilitates the entry of water and oxygen into the cell and that with increasing absorption of nutrients in the seed, thus invigorated seeds can germinate very quickly and produce stronger seedlings. However, further research should be done to investigate the interaction mechanism between $\mathrm{SiO}_{2}$ nano-particles and seed in cellular and molecular levels.

\section{CONCLUSION}

In conclusion, obtained results revealed that the application of $\mathrm{SiO}_{2}$ nanoparticles enhanced seed germination potential in sunflower. An increase in germination parameters by the application of low concentrations of $\mathrm{SiO}_{2}$ nanoparticles may be effective for the growth and yield of crops. Also, the most important traits were germination percentage, energy of germination, promptness index and germination rate which are reflect the rate and power of seed germination and seedling growth in sunflower.

\section{ACKNOWLEDGMENTS}

We wish to thank Dr. Wei-Kai Yan (Eastern Cereal Oilseed Research Center of Agriculture and Agri-Food, Canada) for making available a time-limited version of GGEbiplot as "testBiplotxlsx".

\section{REFERENCES}

AOSA (Association of Official seed Analysis). 1991. Rules for testing seeds. Seed Science and Technology 12: 18-19.

Ashraf M., Foolad M. R. 2005. Pre-sowing seed treatment-a shotgun approach to improve germination, plant growth, and crop yield under saline and non-saline conditions. Advances in Agronomy 88: 223-271.

Ashraf M.Y., Akhtar K., Hussain F., Iqbal J. 2006. Screening of different accessions of three potential grass species from Cholistan desert for salt tolerance. Pakistan Journal of Botany 38: 1589-1597.

Bao-Shan L., Shao-Qi D., Chun-Hui L., Li-Jun F., Shu-Chun Q., Min Y. 2004. Effect of TMS (nanostructured silicon dioxide) on growth of Changbai Larch seedlings. Journal of Forestry Research 15: 138-140.

Basra S.M.A., Farooq M., Wahid A., Khan M. B. 2006. Rice seed invigoration by hormonal and vitamin priming. Seed Science and Technology 34: 753-758.

Dehghani D., Omidi H., Sabaghnia N. 2008. Graphic analysis of trait relations of canola (Brassica napus L.) using biplot method. Agronomy Journal 100: 760-764.

Ellis R. H., Roberts E. H. 1981. The quantification of ageing and survival in orthodox seeds. Seed Science and Technology 9: 373-409.

FAOSTAT. 2012. FAOSTAT. Food and Agricultural Organisation of the United Nations. Available at: http:// faostat.fao.org.

Farooq M., Basra S.M.A., Hafeez K., Ahmad N. 2005. Thermal hardening: a new seed vigour enhancement tool in rice. Acta Botanica Sinica 47: 187-193.

Haghighi M., Afifipour Z., Mozafarian M. 2012. The effect of N-Si on tomato seed germination under salinity levels. Journal of Biological and Environmental Science 6: 87-90.

Harris D., Joshi A., Khan P.A., Gothkar P., Sodhi P. S. 1999. On-farm seed priming in semi-arid agriculture: development and evaluation in maize, rice and chickpea in India using participatory methods. Experimental Agriculture 35: 15-29. 
Iqbal M., Ashraf M. 2007. Seed treatment with auxins modulates growth and ion partitioning in salt-stressed wheat plants. Journal of Integrative Plant Biology 49: 1003-1015.

Liang Y., Sun W., Zhu Y.G., Christie P. 2007. Mechanisms of silicon-mediated alleviation of abiotic stresses in higher plants: a review. Environmental Pollution 147: 422-428.

Ma J. F. 2004. Role of silicon in enhancing the resistance of plants to biotic and abiotic stresses. Soil Science and Plant Nutrition 50: 11-18.

Ma J. F., Yamaji N. 2008. Functions and transport of silicon in plants. Cellular and Molecular Life Sciences 65: 3049-3057.

Mijic A., Liovic I., Zdunic Z., Maric S., Jeromela A. M., Jankulovska M. 2009. Quantitative analysis of oil yield and its components in sunflower (Helianthus annuиs L.). Romanian Agricultural Research 26: 41-46

Nair R., Poulose A. C., Nagaoka Y., Yoshida Y., Maekawa T., Kumar D. S. 2011. Uptake of FITC labeled silica nanoparticles and quantum dots by rice seedlings: effects on seed germination and their potential as biolabels for plants. Journal of fluorescence 21: 2057-2068.

Ranal M. A., Santana, D. G. D. 2006. How and why to measure the germination process?. Brazilian Journal of Botany 29: 1-11.

Ruan S., Xue Q., Tylkowska K. 2002. The influence of priming on germination of rice (Oryza sativa L.) seeds and seedling emergence and performance in flooded soil. Seed Science and Technology 30: 61-67.

Rubio J., Cubero J.I., Martín L.M., Suso M.J., Flores F. 2004. Biplot analysis of trait relations of white lupin in Spain. Euphytica 135: 217-224.

Sabaghnia N., Dehghani H., Alizadeh B., Moghaddam M. 2011. Yield analysis of rapeseed (Brassica napus L.) under water-stress conditions using GGE biplot methodology. Journal of Crop Improvement 25: 26-45.

Sabaghnia N., Janmohammadi M. 2014. Graphic analysis of nano-silicon by salinity stress interaction on germination properties of lentil using the biplot method. Agriculture and Forestry 60: 29-40.

Siddiqui M. H., Al-Whaibi M. H. 2014. Role of nano- $\mathrm{SiO}_{2}$ in germination of tomato (Lycopersicum esculentum seeds Mill.). Saudi Journal of Biological Sciences 21: 13-17.

Wahid A., Noreen A., Basra S. M., Gelani S., Farooq M. 2008. Priming-induced metabolic changes in sunflower (Helianthus annuиs) achenes improve germination and seedling growth. Botanical Studies 49: 343-350.

Yan W. 2001. GGEbiplot-A Windows application for graphical analysis multienvironment trial data and other types of two-way data. Agronomy Journal 93: 1111-1118.

Yan W., Hunt L.A., Sheng Q., Szlavnics Z. 2000. Cultivar evaluation and mega-environment investigation based on the GGE biplot. Crop Science 40: 597-605

Yan W., Kang M S., Ma B., Woods S., Cornelius P.L. 2007. GGE biplot vs. AMMI analysis of genotype-byenvironment data. Crop Science 47: 643-655.

Yan W., Rajcan I. 2002. Biplot evaluation of test sites and trait relations of soybean in Ontario. Crop Science, $42,11-20$.

Yan, W., Hunt L. A. 2002. Biplot analysis of diallel data. Crop Science 42: 21-30. 